\title{
Elaboration D'un Plan de Maîtrise et de Contrôle des Dangers au Cours de la Production de Boisson Gazeuse en Bouteille PET À Partir de la Démarche HACCP
}

\author{
Oumarou Samna Soumana, \\ Université Boubacar Bâ de Tillabéri,
}

Faculté des Sciences Agronomiques, Tillabéri, Niger

Université Dan Dicko Dankoulodo de Maradi (UDDM), Faculté d'Agronomie et des Sciences de l'Environnement, Maradi, Niger

Issoufou Amadou,

Université Dan Dicko Dankoulodo de Maradi (UDDM), Faculté d'Agronomie et des Sciences de l'Environnement, Maradi, Niger

\section{Ali Hama Ibrahim,}

Université Boubacar Bâ de Tillabéri, Faculté des Sciences Agronomiques, Tillabéri, Niger. Université Dan Dicko Dankoulodo de Maradi (UDDM), Faculté d'Agronomie et des Sciences de l'Environnement, Maradi, Niger

\section{Mahamadou Elh. Gounga,}

Université Dan Dicko Dankoulodo de Maradi (UDDM), Faculté

d'Agronomie et des Sciences de l'Environnement, Maradi, Niger

\section{Résumé}

La sécurité sanitaire des aliments est un aspect important à prendre en compte par les entreprises agroalimentaires à travers une démarche qualité afin d'assurer la sécurité des produits alimentaires fabriqués, et garantir par voie de conséquence la santé des consommateurs au Niger. Le présent travail traite de l'élaboration d'un plan de maîtrise et de contrôle à la lumière de l'analyse critique du process de fabrication de l'unité de mise en bouteille de boissons gazeuses, une industrie agroalimentaire du Niger. Les résultats de ce travail apportent des solutions pratiques pour la maîtrise, la prévention et surveillance des 16 points de contrôle critique (PCC) identifiés lors de la fabrication de boissons gazeuses en bouteille PET de cette entreprise. De ce fait un plan de maîtrise des PCC comportant six points de contrôle a été élaboré. Enfin, la mise en œuvre du système HACCP dans ce type entreprise agroalimentaire peut effectivement lui assurer la qualité sanitaire de ses boissons gazeuses PET et élargir son marché. 
Mots clés : Boisson gazeuse PET, HACCP, Analyse des risques, Industries agroalimentaires, Niger

\title{
Elaboration and Planning a Control of Hazards During the Production of Carbonated Beverage in PET Bottle Using the HACCP Approach
}

\author{
Oumarou Samna Soumana, \\ Université Boubacar Bâ de Tillabéri,
}

Faculté des Sciences Agronomiques, Tillabéri, Niger

Université Dan Dicko Dankoulodo de Maradi (UDDM), Faculté d'Agronomie et des Sciences de l'Environnement, Maradi, Niger

\section{Issoufou Amadou,}

Université Dan Dicko Dankoulodo de Maradi (UDDM), Faculté d'Agronomie et des Sciences de l'Environnement, Maradi, Niger

\section{Ali Hama Ibrahim,}

Université Boubacar Bâ de Tillabéri, Faculté des Sciences Agronomiques, Tillabéri, Niger. Université Dan Dicko Dankoulodo de Maradi (UDDM), Faculté d'Agronomie et des Sciences de l'Environnement, Maradi, Niger

\section{Mahamadou Elh. Gounga,}

Université Dan Dicko Dankoulodo de Maradi (UDDM), Faculté d'Agronomie et des Sciences de l'Environnement, Maradi, Niger

\begin{abstract}
Food safety is an important aspect to be taken into account by food companies through a quality approach to ensure the safety of manufactured food products, and thus guarantee the health of consumers in Niger. The present work aims to highlight the development of a plan of control and developed control through the critical analysis of the manufacturing process of the soft drink bottling unit in a food industry in Niger. The results of this work provide practical solutions for the control and prevention of 16 critical control point (CCP) identified during the production of the soft drinks bottling of the company. Thus, a master plan of the CCP with six (6) control points was developed. Finally, implementing the HACCP system in such company can effectively ensure food quality and expand its market.
\end{abstract}


Keywords: Soft Drink, HACCP, Risk analysis, Food Factories, Niger

\section{Introduction}

Les efforts visant à améliorer la qualité et la sécurité des aliments, peuvent être réalisés par une mise en œuvre intégrée cohérente des principes de base des bonnes pratiques d'hygiène (BPH), des bonnes pratiques de fabrication $(\mathrm{BPF})$ et de l'analyse des risques et points critiques pour leur maîtrise ou Hazard Analysis Critical Control Points (HACCP) (Yunus, 2016 ; Surak, 2003) Durant les années 1990, le système HACCP appelé aussi ou ADMPC «Analyse des Dangers-Maîtrise des Points Critiques » a été introduit en tant que mesures obligatoires dans certains secteurs de l'industrie alimentaire (comme le secteur de la viande et des produits laitiers, des produits de la mer) (Cormier et al., 2017). Depuis quelques années, la mise en conformité au système HACCP, souvent dans le cadre volontaire des systèmes de qualité (séries ISO 9000, normes BRC, certifications de produits, etc.), est devenue une norme minimale pour accéder au marché des produits alimentaires (Romano et al., 2004).

Un des systèmes le plus adapté pour les industries agroalimentaires est de loin la méthode HACCP. Cette démarche HACCP reconnue, efficace et préventive garanti l'hygiène des denrées alimentaires, dans le monde entier. À l'heure actuelle, le système HACCP est largement adopté et mis en œuvre par de nombreux pays tels que les États-Unis, le Canada, le Japon, le RoyaumeUni et les États membres de l'Union Européenne, certains pays émergents et en développement ainsi que par des organisations internationales telles que l'OMS, la FAO et surtout la Commission du Codex Alimentarius (Bonne, 2013 ; Dergal, 2015 ; Surak, 2003). En outre, de nombreux pays tels que les États-Unis, le Canada, les pays membres de l'Union européenne, le Japon, etc. ont de plus en plus des exigences strictes en matière d'importation de produits alimentaires (Lu et al., 2014). Devant la globalisation des échanges internationaux que connaît le monde, et pour être crédible et compétitive, la production agroalimentaire requiert un ensemble de contrôle visant à garantir la qualité des produits qu'elle met sur le marché. Ainsi, de nos jours, le souci majeur des entreprises agroalimentaires, qui veulent rester compétitives et exporter leurs produits, n'est plus seulement de fabriquer de produits en grande quantité, mais de tenir compte aussi de l'aspect sécurité. La mise en œuvre du système HACCP dans la fabrication des produits alimentaires peut effectivement garantir la sécurité et la qualité des aliments, élargir le marché et améliorer le niveau de gestion des fabricants (Ropkins \& Beck, 2002).

Pour garantir la sécurité de la production, le système HACCP peut être appliqué tout au long du processus de fabrication, et vu l'importance de la sécurité sanitaire des aliments sur la santé du consommateur, il a été entrepris 
une démarche qualité pour assurer la sécurité des produits au sein d'une des industries agroalimentaires du Niger. Une première étape a d'abord consisté au déploiement des premières phases de la démarche HACCP. Il s'en est suivi le recensement des dangers biologiques, chimiques et physiques pouvant exister à chaque étape de la production (López et al., 2012). Par la suite, les différents points critiques sont identifiés en fonction des limites critiques définies. De plus, pour les points de contrôle critique retenus, la surveillance, les mesures correctives, les mesures préventives, les enregistrements et les vérifications sont établis via l'élaboration d'un plan de maîtrise et de contrôle des dangers au cours de la production de boisson gazeuse en bouteille PET (Quaye, 2018 ; Mortimore \& Wallace, 2013). Ce plan de maîtrise a permis de décrire tous les contrôles à effectuer au cours du processus de production de la boisson gazeuse en bouteille PET. C'est donc un outil de travail mise en place pour : i) analyser et déterminer tous les points critiques (PCC) depuis la réception de la matière première jusqu'au produit fini, ii) proposer un plan de maîtrise des points critiques (PCC) et iii) enfin, proposer un plan de contrôle permettant de mieux garantir la salubrité des aliments produits et mis à la disposition des consommateurs.

\section{Methodologie}

\section{Méthode d'analyse des dangers}

Pour analyser les dangers la méthode utilisée est celle dite des $5 \mathrm{M}$ (Main d'œuvre, Matières premières, Matériels, Milieu, Méthodes), ou diagramme de causes à effets, appelé aussi diagramme d'ISHIKAWA. Généralement, à ce niveau, il faut tout d'abord rechercher les causes de tous les dangers énumérés au moyen de techniques comme l'AMDEC (Analyse des Modes de Défaillances, de leurs Effets et de leur Criticité) (Ryu et al., 2013 ; Wallace et al., 2005 ; Bryan \& WHO,1992). Cela permet ainsi de déterminer les types de dangers auxquels on peut s'attendre et les sources de chaque danger listé et d'en rechercher leurs effets et leur criticité (Tadić et al., 2007). Cette méthode se réfère alors à trois (3) critères : la fréquence, la gravité, la détectabilité, et les risques ont été classés selon leur criticité, tels que le montre le Tableau 1. 
Tableau 2 : Critères d'évaluation des dangers

\begin{tabular}{|c|c|c|}
\hline Critères & Niveau & Signification \\
\hline \multirow[t]{3}{*}{ Fréquence $(\mathrm{F})$} & 1 & Rarement \\
\hline & 3 & Quelques fois \\
\hline & 5 & Souvent \\
\hline Détectabilité & 1 & Danger très facile à détecter \\
\hline \multirow[t]{2}{*}{ (D) } & 3 & Danger facile à détecter \\
\hline & 5 & Danger difficile à détecter \\
\hline \multirow[t]{3}{*}{ Gravité (G) } & 1 & Conséquence faible \\
\hline & 3 & $\begin{array}{l}\text { Conséquence moyenne pouvant laisser des } \\
\text { séquelles non graves }\end{array}$ \\
\hline & 5 & $\begin{array}{l}\text { Conséquence forte pouvant entrainer la mort ou } \\
\text { laisser des séquelles graves }\end{array}$ \\
\hline
\end{tabular}

Sur la base de la valeur attribuée, on calcule la criticité (C) de chaque danger suivant la formule : $\mathbf{C}=\mathbf{F} * \mathbf{G} * \mathbf{D}$.

On définit alors un danger comme mineur si l'indice de criticité $C$ est inférieur ou égale à 15 , majeur si l'indice de criticité $\mathrm{C}$ est supérieur à 15 . Ce seuil est fixé arbitrairement au début de l'étude après des échanges avec les parties prenantes (dont le personnel de l'entreprise) et en se basant sur des expériences antérieures. Il devrait permettre de bien prendre en compte le maximum des étapes sensibles. Les dangers peuvent survenir à chaque étape de la production (Dzwolak, 2014; Bonne, 2013), il faut donc une grande rigueur et une démarche méthodique pour leurs maîtrises. Il existe cinq principales sources de dangers (Matériel, Main d'œuvre, Matière première, Méthode et Milieu) qu'il convient de bien identifier en vue de pouvoir mettre en œuvre les mesures préventives liées à chacune d'entre elles (Pierson, 2012 ; Broutin et al., 2007).

\section{Méthode de détermination des points de contrôle critique}

L'arbre de décision (Figure 1) consiste en une série systématique de quatre questions conçues pour estimer objectivement si un PCC est nécessaire pour maîtriser un danger potentiel identifié à une étape donnée (Codex Alimentarius Commission, 2007). 


\section{Arbre de décision}

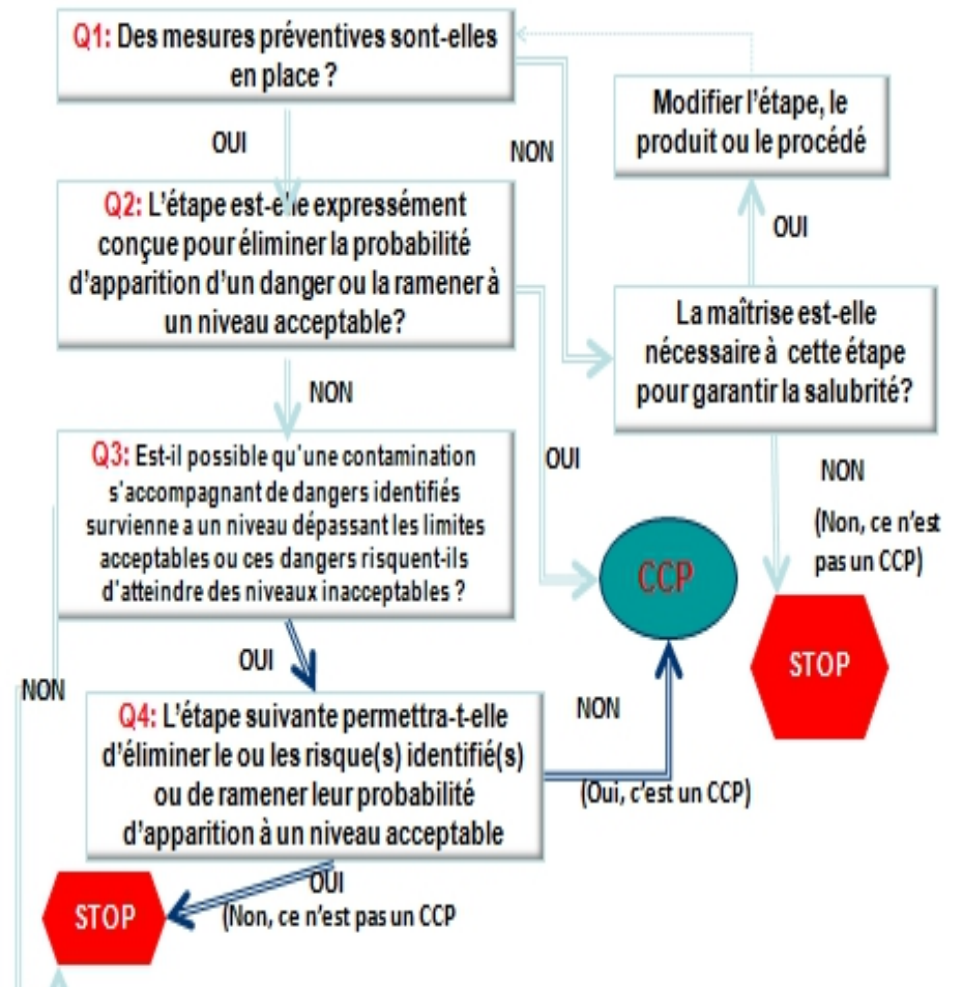

Figure 1 : Arbre de décision adapté du Codex Alimentarius (Codex Alimentarius Commission, 2007)

\section{Resultats}

L'analyse des dangers est une étape clé pour la mise en place de l'autocontrôle de chacun des dangers identifiés (Burstyn et al., 2000). Elle permet de juger des risques pouvant avoir des conséquences négatives sur le consommateur et le producteur. L'évaluation d'un danger potentiel inclut, outre les conséquences possibles, la détermination de sa gravité et de sa fréquence (Ndiaye et al., 2018 ; Tadić et al., 2007). Les différents résultats obtenus au cours de ce travail sont répertoriés dans le tableau d'analyse et d'évaluation des dangers (Tableau 2). 
Tableau 2 : Résultats de l'analyse des dangers

\begin{tabular}{|c|c|c|c|c|c|c|c|}
\hline Etapes & Dangers & Causes & $\begin{array}{l}\text { Facteur } \\
\text { opération } \\
\text { nel }\end{array}$ & & $\begin{array}{c}\text { aluat } \\
\text { D }\end{array}$ & $\mathbf{n}^{\mathrm{C}}$ & Mesures préventives \\
\hline Filtration & $\begin{array}{l}\text { Physiques } \\
\text { Présence de corps étrangers } \\
\text { (sable, pièce métallique) }\end{array}$ & Filtres défectueux & Matériels & 31 & 1 & 3 & $\begin{array}{l}\text { Changer les filtres }(2 / \mathrm{mois}) \\
\text { Vérifier périodiquement les filtres } \\
\text { (1/semaine) } \\
\text { Mirage }\end{array}$ \\
\hline $\begin{array}{l}\text { Désinfection } \\
\text { par UV }\end{array}$ & $\begin{array}{l}\text { Biologique } \\
\text { Présence de germes } \\
\text { pathogènes }\end{array}$ & $\begin{array}{l}\text { Défaillance de la lampe } \\
\text { UV }\end{array}$ & Matériels & 51 & 5 & 25 & $\begin{array}{l}\text { Contrôler périodiquement la lampe UV } \\
\text { (toutes les } 1700 \mathrm{~h} \text { ) } \\
\text { Durée de vie } \leq 7000 \mathrm{~h} \\
\text { Rotation } \geq 25 \mathrm{mj} / \mathrm{cm}^{2}\end{array}$ \\
\hline \multirow{3}{*}{ 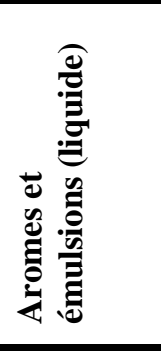 } & $\frac{\text { Physiques }}{\text { Corps étrangers }}$ & Chez le fournisseur & Matières & 31 & 5 & 15 & $\begin{array}{l}\text { Etablir un cahier des charges (évaluation } \\
\text { du fournisseur, fiches de spécifications) }\end{array}$ \\
\hline & $\frac{\text { Chimiques }}{\text { Métaux lourds }}$ & Chez le fournisseur & Matières & 31 & 5 & 15 & $\begin{array}{l}\text { Etablir un cahier de charge (évaluation du } \\
\text { fournisseur, fiches de spécifications) }\end{array}$ \\
\hline & $\begin{array}{l}\text { Biologiques } \\
\text { Flore pathogène }\end{array}$ & $\begin{array}{l}\text { Contamination chez le } \\
\text { fournisseur } \\
\text { Multiplication au cours } \\
\text { de stockage }\end{array}$ & $\begin{array}{l}\text { Matières } \\
\text { et Milieu }\end{array}$ & 51 & 5 & 25 & $\begin{array}{l}\text { Etablir un cahier des charges (évaluation } \\
\text { du fournisseur, fiches de spécifications) } \\
\text { Respecter la température sur l'emballage } \\
\text { (dans tous les cas moins de } 25^{\circ} \mathrm{C} \text { ) }\end{array}$ \\
\hline 吾 & $\frac{\text { Physiques }}{\text { Corps étrangers }}$ & $\begin{array}{l}\text { Au niveau du } \\
\text { fournisseur, Au cours du } \\
\text { transport } \\
\text { Au niveau du magasin de }\end{array}$ & $\begin{array}{l}\text { Matières } \\
\text { et Milieu }\end{array}$ & 11 & 3 & 3 & $\begin{array}{l}\text { Etablir un cahier des charges (évaluation } \\
\text { du fournisseur, fiches de spécifications) } \\
\text { Contrôler à la réception la pureté du sucre }\end{array}$ \\
\hline
\end{tabular}




\begin{tabular}{|c|c|c|c|c|c|c|c|c|}
\hline & $\begin{array}{l}\text { Chimiques } \\
\text { Métaux lourds }\end{array}$ & $\begin{array}{l}\text { Au niveau du fournisseur } \\
\text { Au cours du transport } \\
\text { Au niveau du magasin de } \\
\text { stockage }\end{array}$ & $\begin{array}{l}\text { Matières } \\
\text { et } \\
\text { Milieu }\end{array}$ & 3 & 1 & 5 & 15 & $\begin{array}{l}\text { Etablir un cahier des charges (évaluation } \\
\text { du fournisseur, fiches de spécifications) } \\
\text { Contrôler conformité des matières } \\
\text { premières à la réception } \\
\text { Vérifier à la réception le respect des } \\
\text { paramètres de qualité en fonction du } \\
\text { cahier des charges } \\
\text { Prévoir des magasins adéquats } \\
\text { (entreposage sur des palettes, etc.) }\end{array}$ \\
\hline & $\begin{array}{l}\text { Biologiques } \\
\text { Levures et Moisissures }\end{array}$ & $\begin{array}{l}\text { Contamination au niveau } \\
\text { du fournisseur } \\
\text { Humidité (ERH) } \\
\text { Contamination au cours } \\
\text { du transport } \\
\text { Contamination au niveau } \\
\text { du stockage }\end{array}$ & $\begin{array}{l}\text { Matières } \\
\text { Milieu }\end{array}$ & 3 & 1 & 3 & 9 & $\begin{array}{l}\text { Etablir un cahier des charges (évaluation } \\
\text { du fournisseur, fiches de spécifications) } \\
\text { Contrôler les camions à la réception } \\
\text { Vérifier à la réception le respect des } \\
\text { paramètres de qualité en fonction du } \\
\text { cahier des charges } \\
\text { Prévoir des magasins adéquats } \\
\text { (contrôleur et réducteur d'humidité, } \\
\text { entreposage sur des palettes, etc.) }\end{array}$ \\
\hline \multirow{3}{*}{ 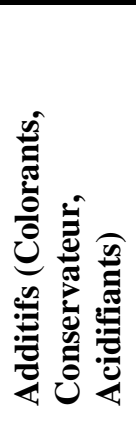 } & $\frac{\text { Physiques }}{\text { Corps étrangers }}$ & Chez le fournisseur & Matières & 3 & 1 & 3 & 9 & $\begin{array}{l}\text { Etablir un cahier des charges (évaluation } \\
\text { du fournisseur, fiches de spécifications) }\end{array}$ \\
\hline & $\frac{\text { Chimiques }}{\text { Présence des métaux lourds }}$ & $\begin{array}{l}\text { Chez le fournisseur } \\
\text { Risque de livraison de } \\
\text { produits non conformes } \\
\text { (non alimentaires) }\end{array}$ & $\begin{array}{l}\text { Matières } \\
\text { et Milieu }\end{array}$ & 3 & 1 & 3 & 9 & $\begin{array}{l}\text { Etablir un cahier des charges (évaluation } \\
\text { du fournisseur, fiches de spécification) }\end{array}$ \\
\hline & $\frac{\text { Biologiques }}{\text { Germes pathogènes }}$ & $\begin{array}{l}\text { Contamination au niveau } \\
\text { du fournisseur }\end{array}$ & Matière & 3 & 1 & 3 & 9 & $\begin{array}{l}\text { Etablir un cahier des charges (évaluation } \\
\text { du fournisseur, fiches de spécification) } \\
\text { Contrôle à la réception (analyse } \\
\text { microbiologique) }\end{array}$ \\
\hline
\end{tabular}




\begin{tabular}{|c|c|c|c|c|c|c|c|c|}
\hline ঠ̃ & $\frac{\text { Chimiques }}{\text { Présence des métaux lourds }}$ & Chez le fournisseur & $\begin{array}{l}\text { Matières } \\
\text { et } \\
\text { Matériels }\end{array}$ & 3 & 1 & 3 & 9 & $\begin{array}{l}\text { Etablir un cahier des charges (évaluation } \\
\text { du fournisseur, fiches de spécification)- } \\
\text { Prévoir un filtre à charbon }\end{array}$ \\
\hline \multirow{2}{*}{ 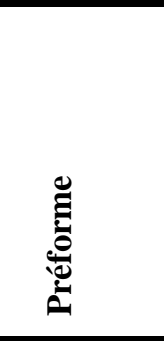 } & $\begin{array}{l}\text { Physiques } \\
\text { Corps étrangers }\end{array}$ & $\begin{array}{l}\text { Contamination chez le } \\
\text { fournisseur } \\
\text { Mauvaise stockage } \\
\text { Négligence par le } \\
\text { personnel }\end{array}$ & $\begin{array}{l}\text { Matières } \\
\text { Milieu et } \\
\text { Main } \\
\text { d'œuvre }\end{array}$ & 3 & 5 & 3 & 45 & $\begin{array}{l}\text { Contrôler à la réception les préformes } \\
\text { Prévoir un magasin propre et à labri de la } \\
\text { poussière } \\
\text { Former le personnel }\end{array}$ \\
\hline & $\frac{\text { Chimiques }}{\text { Présence des métaux lourds }}$ & $\begin{array}{l}\text { Risque de livraison de } \\
\text { produits non alimentaire }\end{array}$ & Matières & 3 & 1 & 5 & 15 & $\begin{array}{l}\text { Etablir un cahier des charges + évaluer le } \\
\text { fournisseur } \\
\text { Contrôle à la réception }\end{array}$ \\
\hline \multirow[b]{3}{*}{ 言 } & $\begin{array}{l}\frac{\text { Physiques }}{\text { Poussières, }} \\
\text { bavures en plastiques }\end{array}$ & $\begin{array}{l}\text { Contamination chez le } \\
\text { fournisseur } \\
\text { Mauvais stockage } \\
\text { Négligence par le } \\
\text { personnel }\end{array}$ & $\begin{array}{l}\text { Matières } \\
\text { Milieu } \\
\text { Main } \\
\text { d'œuvre }\end{array}$ & 3 & 3 & 3 & 27 & $\begin{array}{l}\text { Contrôle à la réception } \\
\text { Prévoir un magasin adéquat } \\
\text { Sensibiliser et Former le personnel }\end{array}$ \\
\hline & $\frac{\text { Chimiques }}{\text { Présence des métaux lourds }}$ & $\begin{array}{l}\text { Risque de livraison de } \\
\text { produit non alimentaire } \\
\text { ou non conforme }\end{array}$ & Matières & 3 & 1 & 5 & 15 & $\begin{array}{l}\text { Etablir un cahier des charges (évaluer le } \\
\text { fournisseur, fiches de spécification) }\end{array}$ \\
\hline & $\frac{\text { Biologiques }}{\text { Coliformes fécaux }}$ & $\begin{array}{l}\text { Chez le fournisseur } \\
\text { Manipulation par les } \\
\text { ouvriers }\end{array}$ & $\begin{array}{l}\text { Matières } \\
\text { Main } \\
\text { d'œuvre }\end{array}$ & 3 & 1 & 3 & 9 & $\begin{array}{l}\text { Etablir un cahier des charges (évaluer le } \\
\text { fournisseur, fiches de spécification) } \\
\text { Sensibiliser et Former le personnel }\end{array}$ \\
\hline \multirow{2}{*}{ 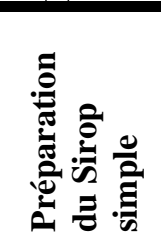 } & $\begin{array}{l}\text { Physiques } \\
\text { Présence de corps étrangers } \\
\text { (Boutons, Pièce métallique, }\end{array}$ & $\begin{array}{l}\text { Matière première } \\
\text { contaminée }\end{array}$ & Matières & 3 & 1 & 3 & 9 & \multirow{2}{*}{$\begin{array}{l}\text { Etablir un cahier des charges (évaluer le } \\
\text { fournisseur, fiches de spécification) } \\
\text { Sensibiliser et Former le personnel } \\
\text { Etablir un Planning et contrôle de la } \\
\text { maintenance préventive }\end{array}$} \\
\hline & jointure) & $\begin{array}{l}\text { Personnel } \\
\text { Usure tuyauterie }\end{array}$ & $\begin{array}{l}\text { Main } \\
\text { d'œuvre } \\
\text { Matériels }\end{array}$ & $\begin{array}{l}1 \\
3\end{array}$ & $\begin{array}{l}1 \\
1\end{array}$ & $\begin{array}{l}1 \\
\mathbf{3}\end{array}$ & $\begin{array}{l}1 \\
9\end{array}$ & \\
\hline
\end{tabular}




\begin{tabular}{|c|c|c|c|c|c|c|c|c|}
\hline & $\begin{array}{l}\frac{\text { Chimiques }}{\text { Métaux lourds }} \\
\text { Traces des produits de } \\
\text { nettoyage et désinfection }\end{array}$ & Usures des équipements & $\begin{array}{l}\text { Matériels } \\
\text { Méthodes } \\
\text { et main } \\
\text { d'œuvre }\end{array}$ & 3 & 1 & 3 & 9 & $\begin{array}{l}\text { Planning et contrôle de la maintenance } \\
\text { préventive } \\
\text { Etablir un cahier des charges (évaluer le } \\
\text { fournisseur, fiches de spécification) } \\
\text { Respecter le plan d'hygiène (GBPH) } \\
\text { Sensibiliser et former le personnel }\end{array}$ \\
\hline & $\begin{array}{l}\frac{\text { Biologiques }}{\text { Coliformes fécaux }} \\
\text { Levures et moisissures }\end{array}$ & $\begin{array}{l}\text { Manipulation du } \\
\text { personnel } \\
\text { Les équipements et } \\
\text { matériaux }\end{array}$ & $\begin{array}{l}\text { Matériels } \\
\text { Main } \\
\text { d'œuvre }\end{array}$ & 5 & 1 & 3 & 15 & $\begin{array}{l}\text { Respecter le plan d'hygiène (GBPH) } \\
\text { Sensibiliser et former le personnel }\end{array}$ \\
\hline : & $\begin{array}{l}\text { Physiques } \\
\text { Corps étrangers (Boutons, } \\
\text { pièce métallique, jointure) }\end{array}$ & $\begin{array}{l}\text { Matière première } \\
\text { Personnel } \\
\text { Usure tuyauterie }\end{array}$ & $\begin{array}{l}\text { Matières } \\
\text { Main } \\
\text { d'œuvre } \\
\text { Matériels }\end{array}$ & 3 & 1 & 3 & 9 & $\begin{array}{l}\text { Etablir un cahier des charges (évaluer le } \\
\text { fournisseur, fiches de spécification), } \\
\text { Sensibiliser et former le personnel } \\
\text { Contrôler la maintenance préventive }\end{array}$ \\
\hline 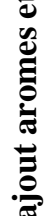 & $\begin{array}{l}\text { Chimiques } \\
\text { Métaux lourds } \\
\text { Trace de produits de } \\
\text { nettoyage et de désinfection }\end{array}$ & $\begin{array}{l}\text { Usures des matériels } \\
\text { Mauvais rinçage }\end{array}$ & $\begin{array}{l}\text { Matériels } \\
\text { Méthodes } \\
\text { et Main } \\
\text { d'œuvre }\end{array}$ & $\begin{array}{l}3 \\
\mathbf{3}\end{array}$ & $\begin{array}{l}1 \\
1\end{array}$ & $\begin{array}{l}3 \\
3\end{array}$ & $\begin{array}{l}9 \\
9\end{array}$ & $\begin{array}{l}\text { Planning et contrôle périodique de la } \\
\text { maintenance préventive } \\
\text { Etablir un cahier de charge (évaluer le } \\
\text { fournisseur, fiches de spécification) }\end{array}$ \\
\hline : & $\begin{array}{l}\text { Biologiques } \\
\text { Coliformes fécaux } \\
\text { Levures et moisissures }\end{array}$ & $\begin{array}{l}\text { Manipulation du } \\
\text { personnel } \\
\text { Les équipements et } \\
\text { matériaux }\end{array}$ & $\begin{array}{l}\text { Matériels } \\
\text { Main } \\
\text { d'œuvre }\end{array}$ & 5 & 1 & 3 & 15 & $\begin{array}{l}\text { Respecter le plan d'hygiène }(\mathrm{GBPH}) \\
\text { Sensibiliser et former le personnel }\end{array}$ \\
\hline
\end{tabular}




\begin{tabular}{|c|c|c|c|c|c|c|c|c|}
\hline 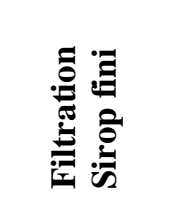 & $\begin{array}{l}\text { Physiques } \\
\text { Corps étrangers (insectes, } \\
\text { araignées, etc.) }\end{array}$ & $\begin{array}{l}\text { Détérioration des filtres, } \\
\text { Mauvais entretien, } \\
\text { Nettoyage insuffisant }\end{array}$ & $\begin{array}{l}\text { Matériels } \\
\text { et main } \\
\text { d'œuvre }\end{array}$ & 51 & & 3 & 15 & $\begin{array}{l}\text { Vérifier et entretenir périodiquement les } \\
\text { filtres }\end{array}$ \\
\hline 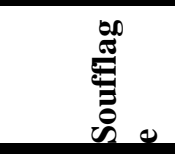 & $\begin{array}{l}\text { Chimiques } \\
\text { Présence d'huile et de } \\
\text { graisse }\end{array}$ & Compresseur à air & Matériels & 1 & 1 & 1 & 1 & $\begin{array}{l}\text { Contrôler et entretenir périodiquement } \\
\text { les filtres }\end{array}$ \\
\hline$\overbrace{0}^{\pi}$ & $\begin{array}{l}\text { Biologiques } \\
\text { Flore bactérienne et } \\
\text { microbienne }\end{array}$ & $\begin{array}{l}\text { Contamination due au } \\
\text { personnel } \\
\text { Air contaminé }\end{array}$ & $\begin{array}{l}\text { Main } \\
\text { d'œuvre } \\
\text { Milieu }\end{array}$ & 3 & 1 & 5 & 15 & $\begin{array}{l}\text { Sensibiliser et former le personnel } \\
\text { Désinfecter les locaux (lors du nettoyage) }\end{array}$ \\
\hline \multirow{2}{*}{ 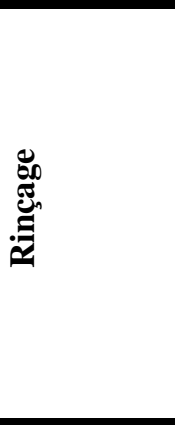 } & $\begin{array}{l}\text { Chimiques } \\
\text { Présence de produits de } \\
\text { Nettoyage }\end{array}$ & $\begin{array}{l}\text { Nettoyage et } \\
\text { Désinfection }\end{array}$ & $\begin{array}{l}\text { Méthodes } \\
\text { et main } \\
\text { d'œuvre }\end{array}$ & 1 & 1 & 1 & 1 & Contrôler l'opération de rinçage (SOP) \\
\hline & $\begin{array}{l}\text { Biologiques } \\
\text { Coliformes fécaux }\end{array}$ & $\begin{array}{l}\text { Manipulation par les } \\
\text { ouvriers } \\
\text { Eau du rinçage } \\
\text { contaminée }\end{array}$ & $\begin{array}{l}\text { Matériels } \\
\text { Matières } \\
\text { Matériels } \\
\text { Méthodes } \\
\text { Main } \\
\text { d'œuvre }\end{array}$ & 3 & 5 & 5 & 75 & $\begin{array}{l}\text { Respecter le plan d'hygiène } \\
\text { Contrôler les lampes UV } \\
\text { Planning de contrôle périodique de la } \\
\text { maintenance } \\
\text { Adapter le système de rinçage } \\
\text { Sensibiliser et former le personnel }\end{array}$ \\
\hline \multirow{2}{*}{ 葛 } & $\begin{array}{l}\text { Physiques } \\
\begin{array}{l}\text { Présence de corps étrangers } \\
\text { (jointures) }\end{array}\end{array}$ & $\begin{array}{l}\text { Détérioration des } \\
\text { jointures }\end{array}$ & Matériels & 5 & 5 & 3 & 75 & $\begin{array}{l}\text { Planning et contrôle périodique de la } \\
\text { maintenance }\end{array}$ \\
\hline & $\begin{array}{l}\text { Chimiques } \\
\text { Traces de produits de } \\
\text { Nettoyage et de Désinfection }\end{array}$ & Mauvais rinçage & $\begin{array}{l}\text { Méthodes } \\
\text { et Main } \\
\text { d'œuvre }\end{array}$ & 3 & 1 & 5 & 15 & Contrôler l'opération de rinçage(SOP) \\
\hline
\end{tabular}




\begin{tabular}{|c|c|c|c|c|c|c|c|c|}
\hline & $\frac{\text { Biologiques }}{\text { Levures et moisissures }}$ & $\begin{array}{l}\text { Mauvaise Nettoyage des } \\
\text { équipements }\end{array}$ & $\begin{array}{l}\text { Matériels } \\
\text { et Main } \\
\text { d'œuvre }\end{array}$ & 3 & 1 & 5 & 15 & $\begin{array}{l}\text { Respecter le plan de Nettoyage et de } \\
\text { désinfection }\end{array}$ \\
\hline \multirow[b]{3}{*}{ : } & $\begin{array}{l}\text { Physiques } \\
\text { Présence de corps étrangers } \\
\text { (jointure, pièce métallique) }\end{array}$ & $\begin{array}{l}\text { Détérioration des } \\
\text { jointures }\end{array}$ & Matériels & 5 & 5 & 3 & 75 & $\begin{array}{l}\text { Planning et contrôle périodique de la } \\
\text { maintenance }\end{array}$ \\
\hline & $\begin{array}{l}\text { Chimiques } \\
\text { Traces de produit de } \\
\text { Nettoyage et de Désinfection } \\
\text { Traces de graisse }\end{array}$ & $\begin{array}{l}\text { Mauvais rinçage } \\
\text { Mauvaise manipulation } \\
\text { du personnel }\end{array}$ & $\begin{array}{l}\text { Méthodes } \\
\text { Main } \\
\text { d'œuvre }\end{array}$ & 3 & 1 & 5 & 15 & $\begin{array}{l}\text { Contrôler l'opération de rinçage } \\
\text { Sensibiliser et former le personnel }\end{array}$ \\
\hline & $\begin{array}{l}\text { Biologiques } \\
\text { Flore bactérienne et } \\
\text { microbienne (Coliformes } \\
\text { fécaux } \\
\text { Germes aérobies, etc.). }\end{array}$ & $\begin{array}{l}\text { Mauvais nettoyage des } \\
\text { équipements } \\
\text { Air contaminé } \\
\text { Manipulation du } \\
\text { personnel }\end{array}$ & $\begin{array}{l}\text { Matériels } \\
\text { Milieu } \\
\text { Main } \\
\text { d'œuvre }\end{array}$ & 5 & 1 & 5 & 25 & $\begin{array}{l}\text { Respecter le plan de Nettoyage et } \\
\text { désinfection } \\
\text { Isoler la soutireuse des autres } \\
\text { équipements } \\
\text { Sensibiliser et former le personnel }\end{array}$ \\
\hline \multirow{2}{*}{ 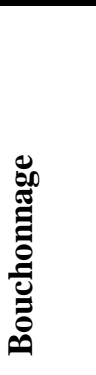 } & $\begin{array}{l}\text { Physiques } \\
\text { Présence de corps étrangers } \\
\text { (Poussières } \\
\text { Pièce métallique) }\end{array}$ & Négligence du personnel & $\begin{array}{l}\text { Main } \\
\text { d'œuvre }\end{array}$ & 3 & 1 & 3 & 9 & $\begin{array}{l}\text { Respecter les conditions de stockage } \\
\text { Sensibiliser le personnel }\end{array}$ \\
\hline & $\frac{\text { Biologiques }}{\text { Coliforme fécaux }}$ & $\begin{array}{l}\text { Manipulation du } \\
\text { personnel }\end{array}$ & Matières & 5 & 1 & 5 & 25 & Traiter et désinfecter par UV \\
\hline
\end{tabular}




\begin{tabular}{|c|c|c|c|c|c|c|c|}
\hline$\sum$ & $\begin{array}{l}\text { Physiques } \\
\text { Présence de corps étrangers } \\
\text { (Bavures, } \\
\text { Pièce métalliques) }\end{array}$ & $\begin{array}{l}\text { Absence de mireur } \\
\text { Absence de miroir }\end{array}$ & $\begin{array}{l}\text { Main } \\
\text { d'œuvre } \\
\text { Matériels }\end{array}$ & 51 & 1 & 5 & $\begin{array}{l}\text { Sensibiliser le personnel } \\
\text { Etudier judicieusement le temps en } \\
\text { fonction de la capacité des agents } \\
\text { faire la rotation des agents } \\
\text { Installer un équipement adapté (miroir) }\end{array}$ \\
\hline 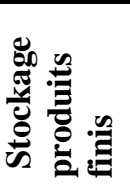 & $\frac{\text { Biologiques }}{\text { Levures et Moisissures }}$ & $\begin{array}{l}\text { Mauvaise condition de } \\
\text { stockage (température } \\
\text { inadéquate) }\end{array}$ & Milieu & 31 & 5 & 15 & $\begin{array}{l}\text { Maîtriser les conditions de stockage } \\
\text { (installer des thermomètres, ventilation/ } \\
\text { aération) }\end{array}$ \\
\hline
\end{tabular}




\section{Les Points de Contrôle Critique (PCC) identifiés}

Il s'agit de déterminer les étapes où les dangers identifiés doivent être maîtrisés. La détermination d'un PCC dans le système HACCP est facilitée principalement par l'application de l'arbre de décision (Figure 1) spécifique à cette démarche (Omer, 2017; Pierson, 2012). L'arbre de décision permet une approche de raisonnement logique. Les résultats de son application dans le cadre de cette étude sont présentés dans le Tableau 3

Tableau 3: Arbre de décision pour la détermination des PCC

\begin{tabular}{cccccccc}
\hline & ETAPES & & Type de & \multicolumn{4}{c}{ DETERMINATION DES PCC } \\
& & Danger & Q1 & Q2 & Q3 & Q4 & Décision \\
\hline $\begin{array}{c}\text { Réception et } \\
\text { stockage de } \\
\text { Matières premières }\end{array}$ & Passage à l'UV & B & Oui & Oui & - & - & PCC 1 \\
& Promes et émulsions & B & Oui & Non & Oui & Non & PCC 2 \\
& & & & & & & \\
\hline & Filtration du sirop simple & P & Oui & Oui & - & - & PCC 5 \\
& Préparation du sirop fini & B & Oui & Non & Oui & Non & PCC 6 \\
& & C & Oui & Non & Oui & Non & PCC 7 \\
& Convoyage & B & Oui & Non & Oui & Non & PCC 8 \\
& Rinçage & B & Oui & Non & Oui & Non & PCC 9 \\
& Premix & B & Oui & Non & Oui & Non & PCC 10 \\
& & P & Oui & Non & Oui & Non & PCC 11 \\
& Soutirage & B & Oui & Non & Oui & Non & PCC 12 \\
& & P & Oui & Non & Oui & Non & PCC 13 \\
& Bouchonnage & B & Oui & Non & Oui & Non & PCC 14 \\
& Mirage & P & Oui & Oui & - & - & PCC 15 \\
& Stockage produit fini & B & Oui & Oui & - & - & PCC 16 \\
\hline
\end{tabular}

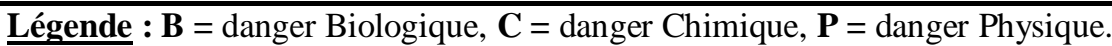

\section{Plan de maîtrise des PCC}

Pour maîtriser les dangers identifiés, un ensemble d'exigences et de documents permettant de justifier la mise en place de bonnes pratiques d'hygiène (BPH) et de laboratoire (BPL) sont établis (DeBeer et al., 2017 ; Mortimore \& Wallace, 2013). Chacune des étapes est traitée dans l'ordre du flux en commençant par les matières premières et en passant par la production jusqu'au stockage (Oscar, 2017 ; Agnide et al., 2015) (Tableau 4). 
Tableau 4: Plan de maîtrise des PCC

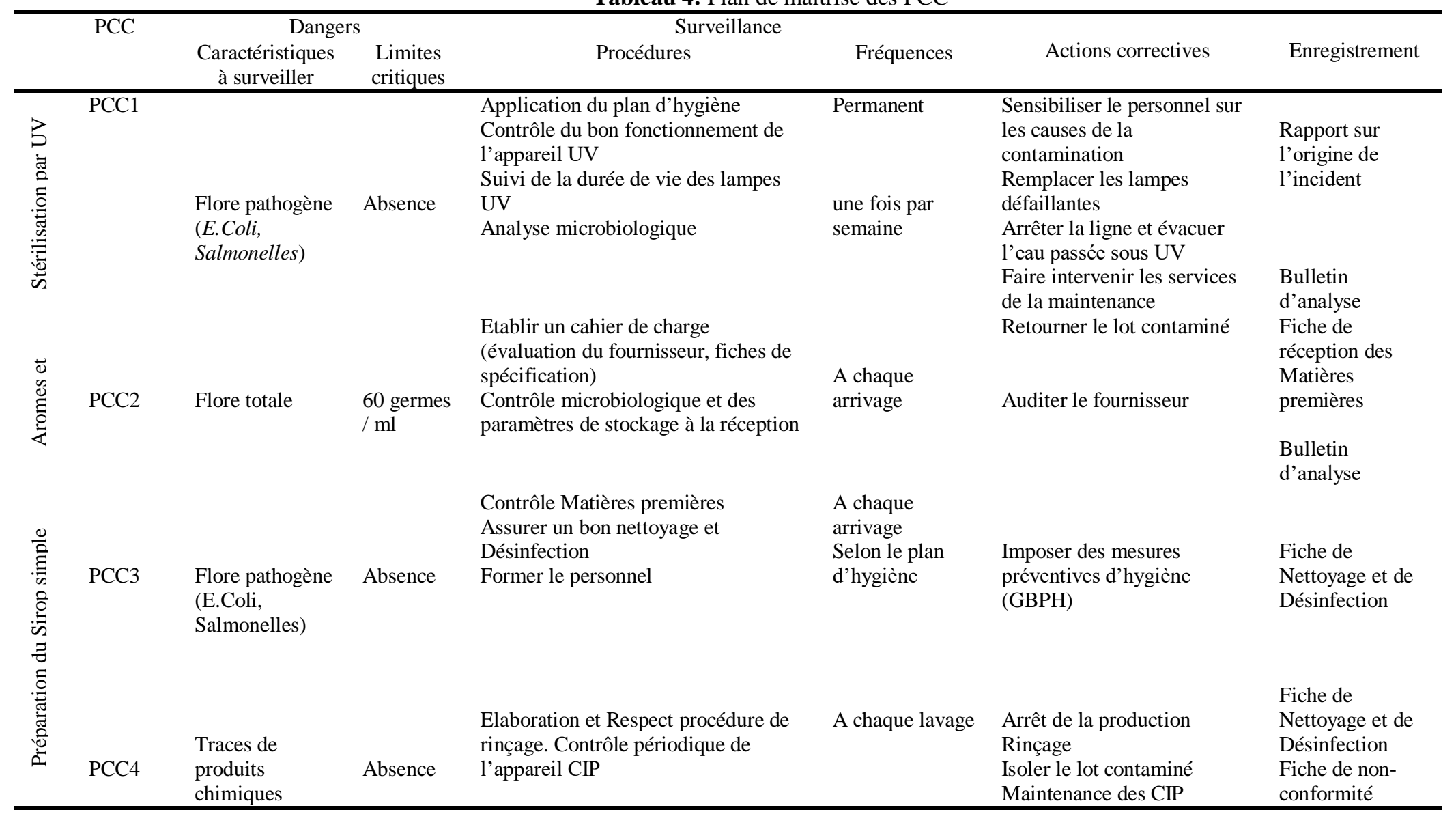




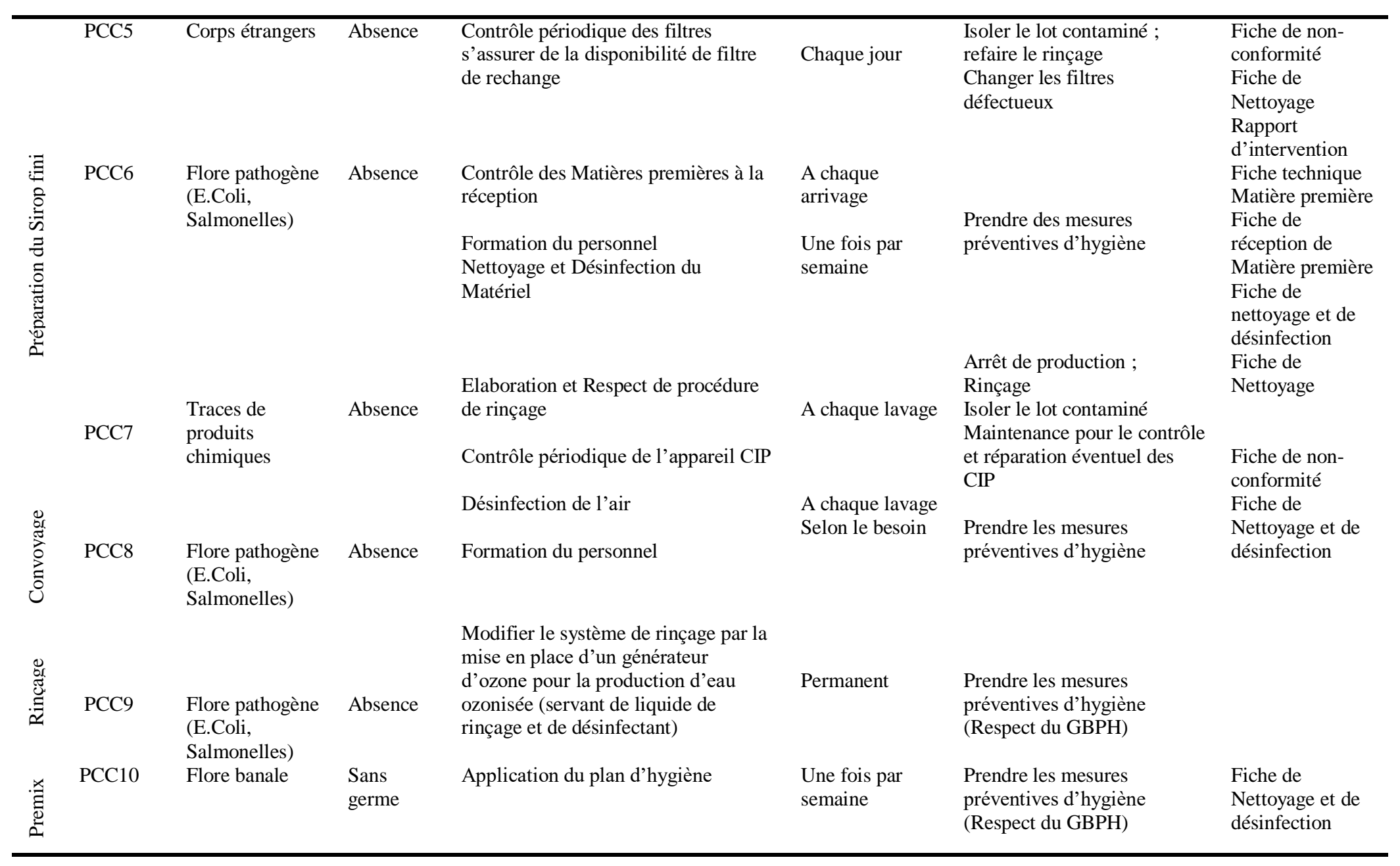




\begin{tabular}{|c|c|c|c|c|c|c|c|}
\hline & PCC11 & $\begin{array}{l}\text { Traces de } \\
\text { produits } \\
\text { chimiques }\end{array}$ & Absence & $\begin{array}{l}\text { Elaboration et Respect de procédure } \\
\text { de rinçage } \\
\text { Contrôle périodique de l'appareil CIP }\end{array}$ & A chaque lavage & $\begin{array}{l}\text { Refaire le rinçage } \\
\text { Faire intervenir la } \\
\text { maintenance pour contrôle et } \\
\text { réparation éventuelle de } \\
\text { l'appareil CIP }\end{array}$ & $\begin{array}{l}\text { Fiche de } \\
\text { Nettoyage et de } \\
\text { désinfection } \\
\text { Rapport sur } \\
\text { l'origine de } \\
\text { l'incident }\end{array}$ \\
\hline & & & & $\begin{array}{l}\text { Application du plan d'hygiène } \\
\text { Formation du personnel }\end{array}$ & $\begin{array}{l}\text { Permanent } \\
\text { Une fois par } \\
\text { semaine }\end{array}$ & $\begin{array}{l}\text { Veiller à l'application stricte } \\
\text { du plan de Nettoyage et de } \\
\text { Désinfection }\end{array}$ & $\begin{array}{l}\text { Fiche de } \\
\text { Nettoyage et de } \\
\text { désinfection }\end{array}$ \\
\hline & PCC12 & $\begin{array}{l}\text { Flore pathogène } \\
\text { (E.Coli, } \\
\text { Salmonelles) }\end{array}$ & Absence & Contrôle du fonctionnement du CIP & A chaque lavage & $\begin{array}{l}\text { Sensibiliser le personnel } \\
\text { Faire intervenir la } \\
\text { maintenance pour contrôle et } \\
\text { réparation de l'appareil CIP }\end{array}$ & $\begin{array}{l}\text { Rapport sur } \\
\text { l'origine de } \\
\text { l'incident }\end{array}$ \\
\hline 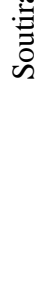 & PCC13 & $\begin{array}{l}\text { Traces de } \\
\text { produits } \\
\text { chimiques }\end{array}$ & Absence & $\begin{array}{l}\text { Elaboration et Respect de procédure } \\
\text { de rinçage } \\
\text { Contrôle du fonctionnement du CIP }\end{array}$ & A chaque lavage & $\begin{array}{l}\text { Arrêter la production; } \\
\text { Rincer } \\
\text { Isoler le lot Contaminé } \\
\text { Faire intervenir la } \\
\text { maintenance pour contrôle et } \\
\text { réparation de l'appareil CIP }\end{array}$ & $\begin{array}{l}\text { Fiche de } \\
\text { Nettoyage et de } \\
\text { désinfection } \\
\text { Fiche de non- } \\
\text { conformité } \\
\text { Rapport sur } \\
\text { l'origine de } \\
\text { l'incident }\end{array}$ \\
\hline 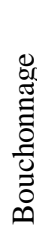 & PCC14 & $\begin{array}{l}\text { Flore pathogène } \\
\text { (E.Coli, } \\
\text { Salmonelles) }\end{array}$ & Absence & $\begin{array}{l}\text { Contrôle de bon fonctionnement de la } \\
\text { bouchonneuse } \\
\text { Application du plan d'hygiène } \\
\text { Sensibilisation du personnel }\end{array}$ & $\begin{array}{l}\text { Chaque jour } \\
\text { Une fois par } \\
\text { Semaine }\end{array}$ & $\begin{array}{l}\text { Faire intervenir la } \\
\text { maintenance } \\
\text { Sensibiliser le personnel }\end{array}$ & $\begin{array}{l}\text { Rapport sur } \\
\text { l'origine de } \\
\text { l'incident } \\
\text { Fiche de } \\
\text { Nettoyage et de } \\
\text { désinfection }\end{array}$ \\
\hline
\end{tabular}




\begin{tabular}{|c|c|c|c|c|c|c|c|}
\hline 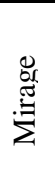 & PCC15 & Corps étrangers & Absence & $\begin{array}{l}\text { Contrôle visuel des bouteilles PET } \\
\text { par mirage à travers un flux lumineux } \\
\text { Rotation des agents de mirage }\end{array}$ & $\begin{array}{l}\text { Permanent } \\
\text { Chaque deux } \\
\text { heures }\end{array}$ & $\begin{array}{l}\text { Isoler le lot contaminé } \\
\text { Sensibiliser le personnel }\end{array}$ & Fiche de contrôle \\
\hline 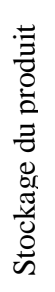 & PCC16 & Flore totale & $\begin{array}{l}60 \text { germes } \\
/ \mathrm{ml}\end{array}$ & $\begin{array}{l}\text { Respecter les conditions de stockage } \\
\text { (stocker dans un lieu frais et propre) } \\
\text { Si la température du magasin } \\
\text { augmente sensiblement prélever un } \\
\text { échantillon pour analyse } \\
\text { bactériologique }\end{array}$ & $\begin{array}{l}\text { Permanent } \\
\text { Une fois par } \\
\text { semaine }\end{array}$ & $\begin{array}{l}\text { Isoler et détruire le lot } \\
\text { contaminé }\end{array}$ & $\begin{array}{l}\text { Rapport Magasin } \\
\text { de Stockage } \\
\text { Fiche de non- } \\
\text { conformité } \\
\text { Bulletin } \\
\text { d'analyse }\end{array}$ \\
\hline
\end{tabular}




\section{Plan de contrôle des PCC}

A l'issue de cette étude, un plan de contrôle a été élaboré afin d'éliminer et/ou de réduire tous les dangers potentiels et d'assurer une bonne gestion de la qualité des produits (Carrascosa et al., 2016). Ce plan est décliné sur la Figure 2.

Figure 2 : Plan de contrôle des dangers potentiels

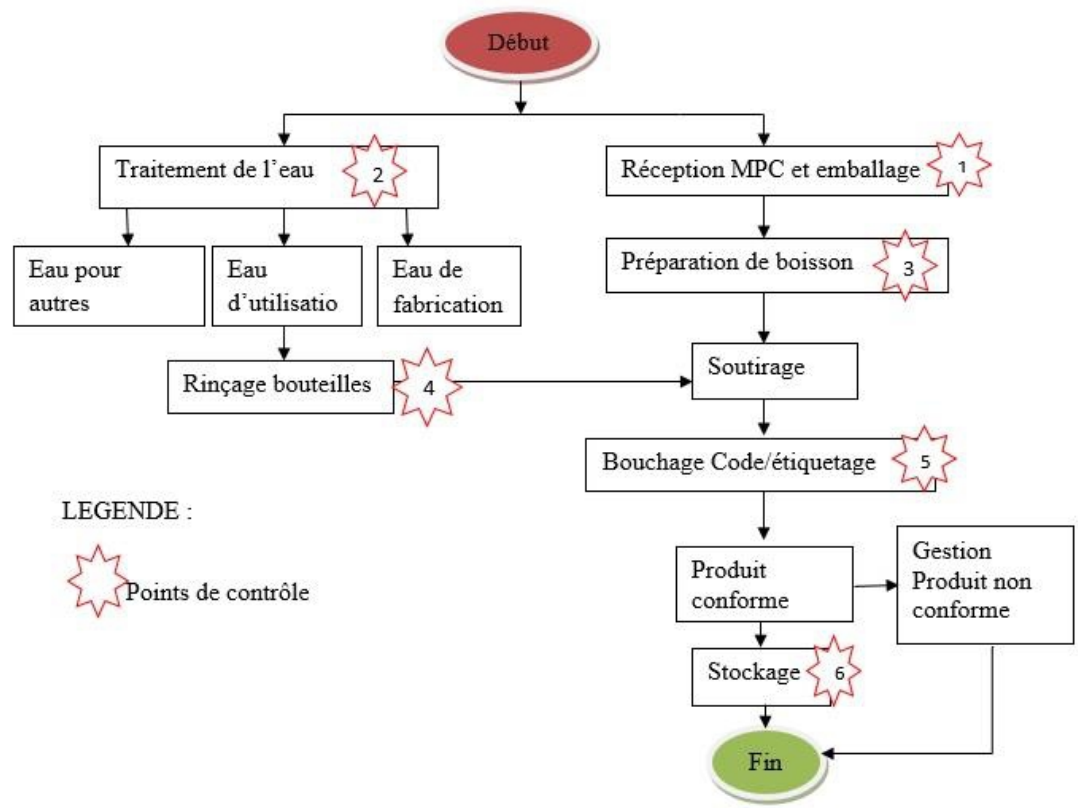

\section{Discussion}

L'analyse des dangers et la détermination des points critiques à maîtriser (PCC) ont été effectuées selon les recommandations du codex alimentarius, les normes de qualité en vigueur (Omer, 2017 ; Al-Busaidi et al., 2017). Ainsi des techniques comme le brainstorming, l'enquête par des questions administrées aux ouvriers et aux chefs d'équipes, et les visites régulières dans la zone de production et les magasins ont permis d'identifier les différents dangers. Ces dangers ont par la suite été classés et évalués en utilisant respectivement la méthode du diagramme Causes-Effets et l'AMDEC. Toutes ces analyses (Tableau 2), couplées à la méthode de l'arbre de décision ont permis de faire ressortir 16 points de contrôle critique dans le process de fabrication (Tableau 3) (Gil Tovar, 2015 ; Mortimore \& Wallace, 2013).

Parmi les 16 points de contrôle critique (PCC) il existe un seul PCC à l'étape de rinçage bouteille vide avant remplissage (PCC9), dont la maîtrise nécessite une modification de système. En effet, le rinçage se fait actuellement à l'eau potable, cette mesure n'est pas suffisante pour éliminer avec efficacité les germes éventuellement présents dans la bouteille. La modification 
proposée pour la maîtrise de ce point critique consiste à utiliser de l'ozone, un puissant oxydant, lors du rinçage de bouteilles. L'installation nécessaire à la production d'ozone n'est pas compliquée mais nécessite l'acquisition d'un générateur d'ozone avec ses accessoires (hotte d'aspiration pour l'évacuation des restes d'ozone à l'extérieur). De plus, une formation à l'utilisation de l'ozone est indispensable. L'application ou la mise en œuvre du plan de maîtrise élaboré permettra d'éliminer ou de réduire à un niveau acceptable les dangers ayant conduit aux 15 autres PCC identifiés lors de l'étude. En somme, les 16 points de contrôle critique identifiés depuis la réception des matières premières jusqu'au produit fini sont localisés principalement au niveau des étapes suivantes: réception de matières premières, $\mathrm{PC}$ et emballage, traitement de l'eau, préparation de boisson, rinçage bouteilles, bouchage et stockage. D'ailleurs, c'est suite à cela que le plan de contrôle a été mis en place avec ses six points de contrôle (Figure 2). Alors, après identification d'une non-conformité suite à un contrôle, une action corrective doit être mise en œuvre de la manière suivante : i) Recherche des causes profondes et des solutions possibles; ii) Choix d'une solution; iii) Mise en œuvre de la solution ; iv) Evaluation de la mise en œuvre ; v) Se référer au Plan de maîtrise (Tableau 4). La maîtrise des PCC atteste qu'une denrée alimentaire est conforme aux caractéristiques spécifiques bien définies.

L'internationalisation des échanges et les exigences des consommateurs sont autant d'enjeux auxquels doivent faire face les acteurs du marché agroalimentaire. Dans ce contexte, un nombre croissant de normes nationales et internationales (dont les exigences réglementaires en vigueur dans la zone UEMOA), reprenant les dispositions des normes ISO qui sont les plus connues, s'imposent comme un standard international en matière d'assurance de la qualité (Pierson, 2012; Surak, 2003) dans les pays africains aussi. La plupart de nos entreprises nationales rencontrent de grandes difficultés pour mettre en place un système de management qualité, ces problèmes sont en général accentués en raison :

$\checkmark$ De la faiblesse des moyens disponibles ;

$\checkmark$ Des difficultés à comprendre les normes et les mettre en application ;

$\checkmark$ Des frais liés à la mise en place et au maintien d'un tel système.

$\checkmark$ Du manque de personnel qualifié dans la mise en place et la gestion des outils de management de la qualité

$\checkmark$ Du manque d'intérêt immédiat pour la société de la mise en place d'une telle démarche.

\section{Conclusion}

Le système HACCP a permis de suivre de l'évolution d'un produit PET pendant toutes les phases de sa fabrication. De plus, un état des lieux des installations, des pratiques de fabrication, de l'hygiène et des contrôles a été 
réalisé. Ce travail a aussi été l'occasion de proposer des améliorations, notamment sur l'aménagement et les agencements des flux ainsi que les moyens de contrôle. La mise en œuvre des bonnes pratiques afin de pouvoir mettre en place le système HACCP a été réalisée. La rédaction et l'application effective des procédures et instructions pour la maitrise des actions préventives est d'une très grande utilité. Enfin, la mise en place du système HACCP permettant principalement de garantir la salubrité et la qualité des aliments est une réelle opportunité pour une entreprise agroalimentaire car elle est très souvent porteuse d'autres avantages sur le plan concurrentiel et financier.

\section{References:}

1. Yunus, M. R. (2016). Hazard Analysis and Critical Control Points in cocoa bean fermentation. International Journal of Agriculture System, 4(1), 13-26.

2. Surak, J. G. (2003). HACCP and ISO development of a food safety management standard. Department of Science and Human Nutrition, 224.

3. Cormier, R. J., Mallet, M., Chiasson, S., Magnússon, H., \& Valdimarsson, G. (2007). Effectiveness and performance of HACCPbased programs. Food Control, 18(6), 665-671.

4. Romano, D., Cavicchi, A., Rocchi, B., \& Stefani, G. (2004). Costs and benefits of compliance for HACCP regulation in the Italian meat and dairy sector (No. 731-2016-50637).

5. Bonne, R. (2013). «La gestion globale de l'hygiène dans les IAA»: une méthode de facilitation pour la mise en œuvre des prescriptions d'hygiène du codex alimentarius. Bulletin de l'Académie Vétérinaire de France.

6. Dergal, N. B. (2015). Evaluation des systèmes de management de la sécurité et de la qualité de l'aquaculture du tilapia du Nil" Oreochromis niloticus" dans l'Ouest algérien (Doctoral dissertation, Université de Liège, Liège, Belgique).

7. Lu, J., Pua, X. H., Liu, C. T., Chang, C. L., \& Cheng, K. C. (2014). The implementation of HACCP management system in a chocolate ice cream plant. Journal of food and drug analysis, 22(3), 391-398.

8. Ropkins, K., \& Beck, A. J. (2002). Application of hazard analysis critical control points (HACCP) to organic chemical contaminants in food. Critical reviews in food science and nutrition, 42(2), 123-149.

9. López, P. D. S., Sandia, M. G., Bou, L. R., \& Hernández, P. S. (2012). Design of an HACCP program for a cocoa processing facility. Archivos latinoamericanos de nutricion, 62(4), 355-362. 
10. Quaye, S. (2018). Development of a HACCP Plan for a Model Cold Pressed Virgin Coconut Oil Industry in Ghana (Doctoral dissertation).

11. Mortimore, S., \& Wallace, C. (2013). HACCP: A practical approach. Springer Science \& Business Media.

12. Ryu, K., Park, K. H., Yang, J. Y., \& Bahk, G. J. (2013). Simple approach in HACCP for evaluating the risk level of hazards using probability distributions. Food control, 30(2), 459-462.

13. Wallace, C. A., Powell, S. C., \& Holyoak, L. (2005). Development of methods for standardised HACCP assessment. British Food Journal, 107(10), 723-742.

14. Bryan, F. L., \& World Health Organization. (1992). Hazard analysis critical control point evaluations: a guide to identifying hazards and assessing risks associated with food preparation and storage.

15. Tadić, D., Stefanović, M., \& Milanović, D. (2007). Fuzzy approach in evaluation of operations in food production. International Journal for Quality Research, (2), 97-104.

16. Dzwolak, W. (2014). HACCP in small food businesses-The Polish experience. Food control, 36(1), 132-137.

17. Pierson, M. D. (2012). HACCP: principles and applications. Springer Science \& Business Media.

18. Broutin, C., François, M., \& Niculescu, N. L. N. (2007). Gestion de la qualité dans la transformation laitière: expérimentation d'une démarche d'élaboration concertée de guides de bonnes pratiques d'hygiène au Sénégal et au Burkina Faso. Revue d'élevage et de médecine vétérinaire des pays tropicaux, 60(1-4), 163-169.

19. Codex Alimentarius Commission, Joint FAO/WHO Food Standards Programme, \& World Health Organization. (2007). Codex alimentarius Commission: procedural manual. Food \& Agriculture Organisation.

20. Burstyn, I., Kromhout, H., Cruise, P. J., \& Brennan, P. (2000). Designing an international industrial hygiene database of exposures among workers in the asphalt industry. The Annals of occupational hygiene, 44(1), 57-66.

21. Ndiaye, N. A., Cisse, M., Bonne, R., Sene, B., Kane, N. C., \& Montet, D. (2018). Application of a facilitating HACCP system using two innovative methods for the production of\# Hibiscus syrup\# by a Senegalese small and medium business (SMBs). International Food Research Journal, 25(1), 376-682.

22. Omer, H. M. A. (2017). Implementing the Hazard Analysis Critical Control Points (HACCP) System in Fish Markets in Khartoum State, Sudan (Doctoral dissertation, Sudan University of Science \& Technology). 
23. DeBeer, J., Nolte, F., Lord, C. W., Colley, J., \& Weddig, L. (2017). Setting HACCP critical limits for the precooking PCC of commercially processed tuna. Food Protection Trends, 37(3), 176-188.

24. Oscar, T. P. (2017). Neural network models for growth of Salmonella serotypes in ground chicken subjected to temperature abuse during cold storage for application in HACCP and risk assessment. International Journal of Food Science \& Technology, 52(1), 214-221.

25. Agnide, M. B., Avoha, B. L., Ahoussi, E., \& Aladjodjo, d. d. (2015). Installation du Systeme HACCP dans une industrie Agro-Alimentaire. EPAC/UAC.

26. Carrascosa, C., Millán, R., Saavedra, P., Jaber, J. R., Raposo, A., \& Sanjuán, E. (2016). Identification of the risk factors associated with cheese production to implement the hazard analysis and critical control points (HACCP) system on cheese farms. Journal of dairy science, 99(4), 2606-2616.

27. Al-Busaidi, M. A., Jukes, D. J., \& Bose, S. (2017). Hazard analysis and critical control point (HACCP) in seafood processing: An analysis of its application and use in regulation in the Sultanate of Oman. Food control, 73, 900-915.

28. Gil Tovar, H. (2015). La gestion de compétences comme stratégie pour la productivité: cas: l'enjeu productif du secteur des passiflores du Département du Huila (Doctoral dissertation, Le Mans). 